\title{
A New Multi-Sensor Target Recognition Framework based on Dempster-Shafer Evidence Theory
}

\author{
Kan Wang* \\ Southwest China Institute of Electronic Technology, CETC Intelligent Joint intelligence Key Laboratory, Chengdu, 610036, China
}

\begin{abstract}
In order to meet the higher requirements in military technology, automation, and intelligence, increasingly importance has been attached to the information fusion for multi-sensor systems. Dempster-Shafer evidence theory is a typical method of uncertainty information fusion due to its adjustability in uncertainty modeling; whereas classical evidence theory is still insufficient in solving high-conflict problems. This assumption studies the multi-sensor information fusion model based on evidence theory from the following aspects. First, it introduces the basic principles of evidence theory, and focuses on how to use triangular fuzzy numbers to obtain basic probability assignments. Second, the method of weighting the evidence using the reliability of the sensor is introduced. The reliability of the sensor is divided into two parts: static reliability and dynamic reliability. Moreover, this model proposes the irrationality of Deng's entropy weight for the binary target recognition problem, and improves the entropy weight in sensor dynamic weights. Finally, on the basis of the above research, sensor sensing data is applied to this model. Through simulation experiments, the validity of the model is proved and the target can be accurately identified.
\end{abstract}

Keywords: information fusion; evidence theory; target recognition; multi-sensor system

(Submitted on February 25, 2018; Revised on April 4, 2018; Accepted on May 3, 2018)

(C) 2018 Totem Publisher, Inc. All rights reserved.

\section{Introduction}

As information technology is rapidly developing and the urgent requirements of military and civilian use, multi-sensor fusion technology has gradually been taken seriously and has been rapidly developed. Because the data collected by a single sensor is often limited and unreliable, it cannot meet the high demand that is gradually rising in practical applications. Multi-sensor information fusion technology overcomes the uncertainties and limitations of a single sensor by multiple fusion processing of information obtained by multiple sensors, and improves the effective performance of the system, thereby obtaining a more accurate result than a single sensor measurement. In the multi-sensor information acquisition system, since the system cannot completely eliminate the negative effect brought by a series of factors such as sensor accuracy, transmission error, environmental noise, and human interference, the obtained data will generate uncertainty [1,2].

The problems that arise in multi-sensor information fusion systems can be summarized as uncertain inference problems [3]. The process of information fusion is actually a process of uncertainty reasoning and decision making. Therefore, how to integrate uncertain information and obtain accurate and effective decision-making results has become the focus of research. As multi-sensor information fusion system is developing rapidly, decision fusion of target recognition has gradually become a hot topic for experts and scholars at home and abroad, especially in aerospace engineering [4], target identification tracking [5-7], fault diagnosis [8-12], image fusion [13-16] and other fields. Therefore, researching target recognition and decision fusion has important theoretical significance and significant practical application value[17,18]. It also has important strategic and social benefits for China's national defense construction.

\footnotetext{
* Corresponding author.

E-mail address: 306616278@qq.com
} 


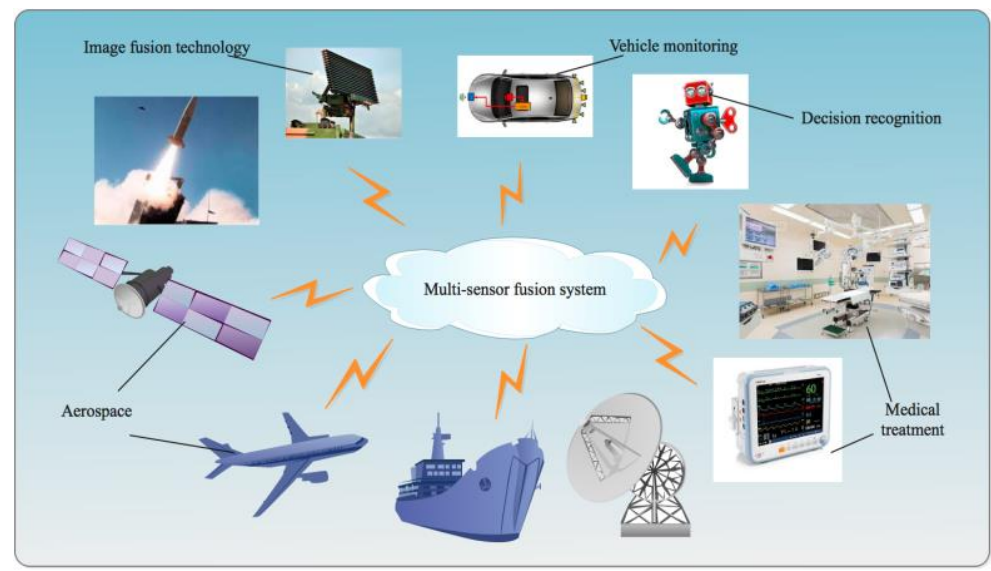

Figure 1. Application of multi-sensor fusion system

At present, decision-making multi-sensor target recognition fusion methods include: target recognition fusion based on maximum posterior probability, target recognition fusion based on Dempster-Shafer (D-S) evidence theory, target recognition fusion based on fuzzy synthesis, and target recognition fusion based on blackboard model. The multi-sensor information fusion algorithm based on D-S evidence theory does not need to specify the prior probability, and can effectively solve the fusion problem caused by the uncertainty information between sensors. It can reasonably process complementary and redundant information to make it easier to make decisions. The result of the synthesis reduces the uncertainty of the system, making the recognition result more accurate and more robust. Therefore, this paper will choose evidence theory as a method of information fusion for multi-sensor systems.

\section{The basic principle of evidence theory}

Evidence theory first defines a non-empty finite set $\Theta$ as recognition framework. $\Theta$ contains $M$ mutually exclusive and exhaustive propositions, $\Theta=\left\{A_{1}, A_{2}, \ldots, A_{M}\right\}$. Among them, $M$ is the total number of propositions in the recognition framework, and $A_{i}(i=1,2, \ldots, M)$ represents the $\mathrm{i}$-th proposition of the recognition framework. A subset of the recognition framework belongs to its power set, denoted as $2^{\Theta} . \varnothing$ is an empty set, then:

$$
2^{\Theta}=\left(\varnothing, H_{1}, H_{2}, \ldots, H_{M},\left\{H_{1}, H_{2}\right\},\left\{H_{1}, H_{3}\right\},\left\{H_{1}, H_{M}\right\}, \ldots,\left\{H_{1}, H_{2}, \ldots, H_{M}\right\}\right)
$$

Assume that $\Theta$ is a complete set and $A$ is a subset of $\Theta$. If function $m: 2^{\Theta} \rightarrow[0,1]$ satisfies the conditions as follows.

$$
\begin{aligned}
& m(\varnothing)=0 \\
& \sum_{A \subseteq \Theta} m(A)=1
\end{aligned}
$$

Then, formula $m(A)$ is called the basic probabilistic assignment function of proposition $A$ on $\Theta$. It is also called Basic Probability Assignment (BPA). It describes the degree of the support of evidence for proposition $A$, and does not support any truthfulness of $A$. set. If $m(A)>0$, then $A$ is called coke.

Assuming that in the same recognition frame $\Theta$, the $N$ evidence bodies after multi-sensor acquisition and processing are $m_{1}, m_{2}, \ldots, m_{N}$, and the focal elements are $A_{1}, A_{2}, \ldots, A_{M}$ respectively, and the evidence bodies are not completely in conflict and independent, then the fusion rule of $N$ evidence bodies is shown as (3).

$$
m(A)= \begin{cases}\frac{1}{1-K} \sum_{\cap A_{i}=A} \prod_{1 \leq k \leq N} m_{k}(A) & A \neq \varnothing \\ 0 & A=\varnothing\end{cases}
$$


$K$ represents the conflicting factor produced in the synthesis process, and equation (4) gives the definition of the conflicting factor.

$$
K=\sum_{\cap A_{i}=\varnothing} \prod_{1 \leq k \leq N} m_{k}\left(A_{i}\right)
$$

\section{Evidence BPA modeling and solution}

The establishment of the propositional representation model under the identification framework based on the existing sample data is an important part of generating BPA. In the mathematical model of the propositional representation under the identification framework, the condition that can be used is a certain amount of data samples; so, the use of extended triangular fuzzy numbers is considered.

The minimum attribute value MIN(A), average attribute value AVE(A), and maximum attribute value MAX(A) of velocity characteristic data A are calculated by statistical samples, and a corresponding triangular fuzzy number is constructed. The height of the fuzzy number is 1 . Since a certain property of the sample data to be measured is a value, a standard triangular fuzzy number cannot be constructed, and this value can be regarded as a special triangular fuzzy number; that is, a line segment whose height is 1 , and its abscissa is its measurement value. After obtaining the triangular fuzzy numbers describing the characteristics of each category, the position of the special segment in each triangle can be used to determine the intersection of the sample to be measured and each triangle. The value of this intersection is the basic probability assignment of the sample to each target. as shown in Figure 2.

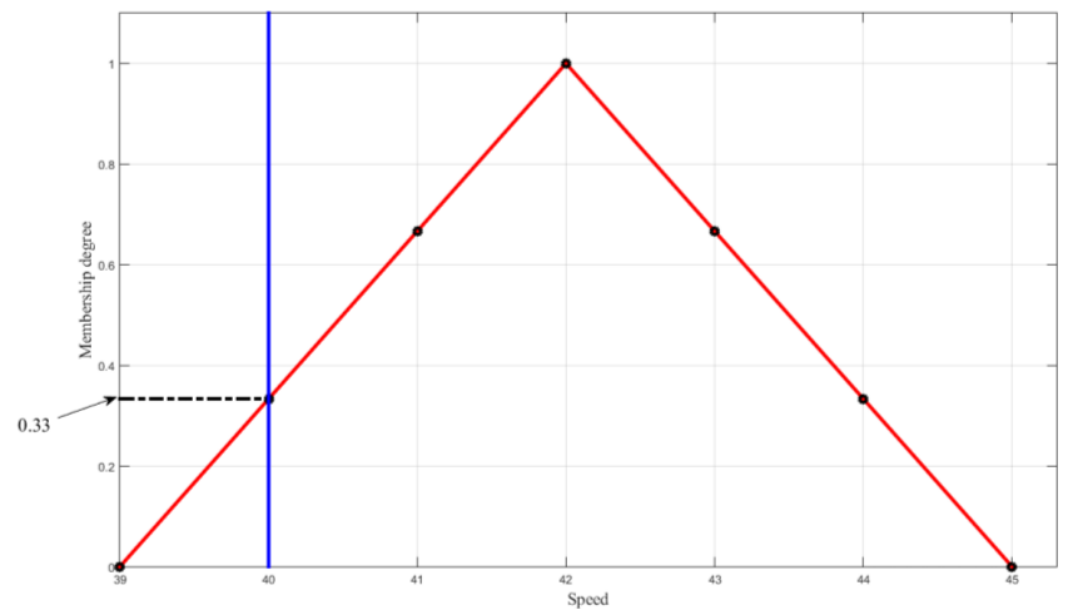

Figure 2. The time-fuzzy number of the sample to be measured

After obtaining the basic probabilistic assignments of the samples to be tested for each category, according to the principles of D-S evidence theory, the sum of the basic probabilities of all subsets is 1 . When the sum of all possibilities of the category they belong to is greater than one, these possibilities are normalized to obtain a general reliability assignment for this set of data. Among them, the normalization process uses $x_{i}=\frac{x_{i}}{\sum_{i=1}^{n} x_{i}}, i=1,2, \ldots, n$ for calculation. When the sum of all possibilities is less than 1 , we assign the sum of all possibilities to the difference of 1 to the set. $m(\Theta)=1-\sum_{A \subset \Theta} m(A)$ and the general confidence assignment of this set of data is made up of the various possibilities and the values of set $\Theta$.

\section{Evidence fusion model establishment and solution}

\subsection{Evidence fusion principle model}

\subsubsection{Weighted fusion method}

The validity of D-S evidence theory in the treatment of uncertain information and data fusion is not questioned. 
However, when dealing with high-conflict evidence, there is often a paradox. Zadeh gives an example of this value [19]:

$$
\begin{aligned}
& m_{1}\left(\left\{F_{1}\right\}\right)=0.9, m_{1}\left(\left\{F_{2}\right\}\right)=0, m_{1}\left(\left\{F_{3}\right\}\right)=0.1 \\
& m_{2}\left(\left\{F_{1}\right\}\right)=0, m_{2}\left(\left\{F_{2}\right\}\right)=0.9, m_{1}\left(\left\{F_{3}\right\}\right)=0.1
\end{aligned}
$$

After blending with formula (3), the BPA value of proposition $F_{3}$ is:

$$
m\left(\left\{F_{3}\right\}\right)=\frac{0.1 \times 0.1}{1-0.9 \times 0.1-0.1 \times 0.9-0.9 \times 0.9}=1
$$

Although both sets of evidence have very low support for propositions, the D-S fusion results have a confidence level of 1 for Proposition $F_{3}$. Obviously, this result is unrealistic and may eventually lead to wrong decisions. To deal with this type of problem, distance measures measure the distance between the evidence by calculating the distance between the evidence, and express the evidence as a vector. The Euclidean distance $d_{i j}$ between the two sets of evidence $m_{1}(\cdot)$ and $m_{2}(\cdot)$ is defined as follows:

$$
d_{i j}=d\left(m_{i}, m_{j}\right)=\left(\sum_{k=1}^{M}\left|m_{i}\left(A_{k}\right)-m_{j}\left(A_{k}\right)\right|^{2}\right)^{1 / 2}
$$

After calculation, a distance matrix is expressed as EDM (Euclidean distance matrix):

$$
E D M=\left(\begin{array}{cccc}
0 & d_{12} & \ldots & d_{1 N} \\
d_{21} & 0 & \ldots & d_{2 N} \\
\ldots & \ldots & \ldots & \ldots \\
d_{N 1} & d_{N 2} & \ldots & 0
\end{array}\right)
$$

The distance measure and similarity between evidence bodies are mutually opposite concepts. That is, the smaller the distance, the greater the similarity between them, and vice versa. Therefore, the correlation between any two sets of evidence bodies $m_{i}$ and $m_{j}$ is defined as follows.

$$
\operatorname{sim}\left(m_{i}, m_{j}\right)=1-d_{i j}
$$

\subsubsection{Deng entropy}

Deng in the literature [20] generalized the Shannon entropy and applied it to the uncertainty measure of evidence. Its defined Deng entropy is represented by equation (10).

$$
E_{d}=-\sum_{i} m\left(A_{i}\right) \log \frac{m\left(A_{i}\right)}{2^{\left|A_{i}\right|}-1}
$$

Where $A_{i}$ is a proposition in the body of evidence and $\left|A_{i}\right|$ is the base of proposition $A_{i}$. When the proof bodies are all single focal elements, the Deng entropy can be expressed in the form of formula (11).

$$
E_{d}=-\sum_{i} m\left(A_{i}\right) \log \frac{m\left(A_{i}\right)}{2^{\left|A_{i}\right|}-1}=-\sum_{i} m\left(A_{i}\right) \log m\left(A_{i}\right)
$$

\subsection{Improved evidence Fusion}

\subsubsection{Joint reliability weighting}

The reliability of the sensor is an important criterion for measuring a sensor. Whether or not the fusion result is reasonable is closely related to the static reliability and dynamic reliability of the sensor, such as accuracy, work efficiency, 
environmental noise, presence of unknown targets, and fraudulent behavior of the observation target [21].

In this paper, the confidence of the target recognition of the sensor $\alpha_{i}(\cdot)$ and the reliability of the sensor $\beta_{i}(\cdot)$ are used to measure the static reliability index of the sensor, the distance [22] between the evidence and the dynamic reliability index of the Deng entropy [23] measurement sensor.

$\omega^{s}$ indicates staic reliability. $\omega^{s}$ is defined as follows.

$$
\omega_{i}^{s}=\alpha_{i} \times \beta_{i}
$$

$\omega^{d}$ indicates the dynamic reliability of the sensor, $\omega^{d}$ is defined as follows.

$$
\omega_{i}^{d}=c r d_{i} \times E d_{i}
$$

Among them, $c r d_{i}$ is calculated by distance, and $E d_{i}$ is calculated by formula (14) normalized.

$$
E d_{i}=\frac{E d(i)}{\max E d(i)}
$$

We synthetically model the reliability of sensors and combine static reliability with dynamic reliability. The definition of the joint weight $\omega$ is as follows.

$$
\omega_{i}=\omega_{i}^{s} \times \omega_{i}^{d}=\alpha_{i} \times \beta_{i} \times c r d_{i} \times E d_{i}
$$

The weight of evidence can be calculated based on the reliability of sensors. Assuming that there is group $n$ evidence, the final weight of the formula (16) normalized is as follows.

$$
\omega_{i}=\frac{\omega(i)}{\sum_{i=1}^{n} \omega(i)}
$$

We use the weights of evidence obtained to correct the original BPA matrix, as shown in equation (17).

$$
m^{\prime}\left(A_{k}\right)=\sum_{i=1}^{N} \omega_{i} m_{i}\left(A_{k}\right) \quad k=1,2, \ldots, M
$$

So far, the $n$ evidence bodies under the recognition framework $\Theta$ have been corrected by weight coefficient $\omega_{i}$ and a new BPA has been obtained. We use DS synthesis rule to synthesize and express it in formula (18).

$$
m(A)=\left\{\begin{array}{cl}
\frac{\sum_{\cap A_{k}=A} \prod_{i=1}^{N} m_{i}^{\prime}\left(A_{k}\right)}{1-\sum_{\cap A_{k}=\varnothing} \prod_{i=1}^{N} m_{i}^{\prime}\left(A_{k}\right)} & A \neq \varnothing, \forall A_{k} \subseteq \varnothing \\
0 & A=\varnothing
\end{array}\right.
$$

The flow chart of the joint weighting algorithm is shown in Figure 3. 


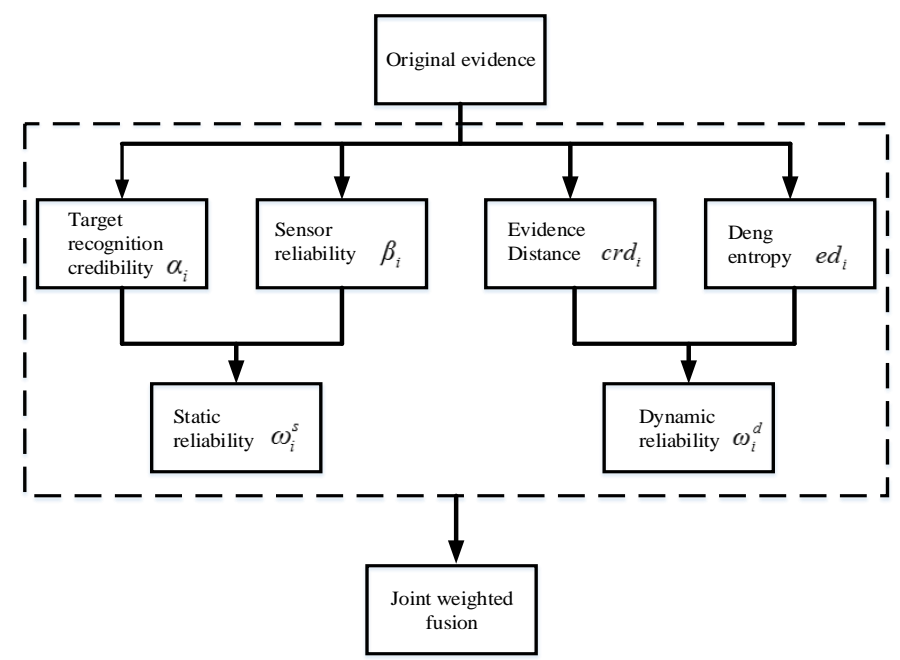

Figure 3. Joint weighted algorithm flow chart

\subsubsection{Improvement of Deng entropy weight for binary identification problem}

If the judgment is made on the recognisability of a target, the evidence identification framework is defined as

$$
\Theta=\{\text { Target } 1 \text { is identifiable, Target } 1 \text { is not identifiable }\}
$$

This problem becomes a binary identification problem. The binary identification Deng entropy curve is shown in Figure 4(a). By observing the binary identification Deng entropy graph, we can see that due to the binary entropy characteristics, after the BPA value of the target to be identified is greater than 0.5, its entropy weight starts to decrease. This is inconsistent with the fact that the greater the BPA value in the evidence theory represents the greater the degree of confidence given.

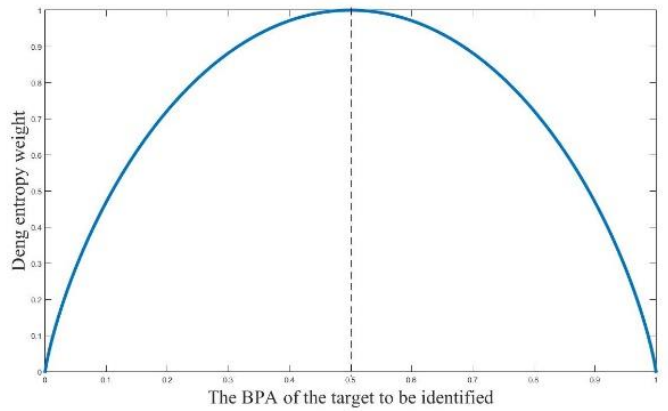

(a)

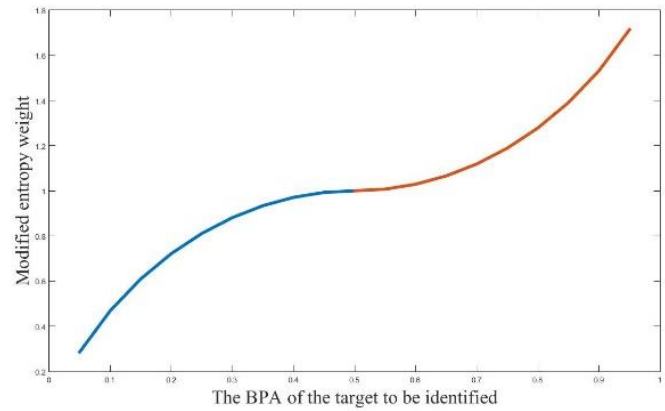

(b)

Figure 4. (a) Binary identification Deng entropy curve (b) Binary recognition modifying entropy weight graph

Therefore, in this paper, the Deng entropy weight is modified for the binary identification problem, and the binary identification modification entropy weight curve is shown in Figure 4(b). The specific calculation formula is formula (20), and the entropy value is calculated according to the growth segment of BPA. In this way, the theory that the greater the entropy weight given by the greater degree of evidence confidence is met.

$$
E_{d}^{\prime}=\left\{\begin{array}{ll}
-\sum_{i} m\left(A_{i}\right) \log \frac{m\left(A_{i}\right)}{2^{\left|A_{i}\right|}-1} & m\left(A_{i}\right)<0.5 \\
2+\sum_{i} m\left(A_{i}\right) \log \frac{m\left(A_{i}\right)}{2^{\left|A_{i}\right|}-1} & m\left(A_{i}\right) \geq 0.5
\end{array} \quad i=1,2 .\right.
$$




\section{Multi-sensor data fusion model}

Assuming there are $k$ sensors, they can be the same type of sensors, or they can be different types of sensors. The identified target belongs to a set of $n$ known target types. Each sensor is estimated based on its own observations, for example, the recognition confidence $\alpha_{k}(j), \alpha_{k}(j) \in[0,1], j=1,2, \ldots, m$ and sensor work reliability $\beta_{k} \in[0,1], k=1,2, \ldots, K$ of the $j$-th sensor for the kth sensor. This estimate is then sent to the target recognition fusion center to make a global estimate of the target recognition based on partial estimates of the k sensors.

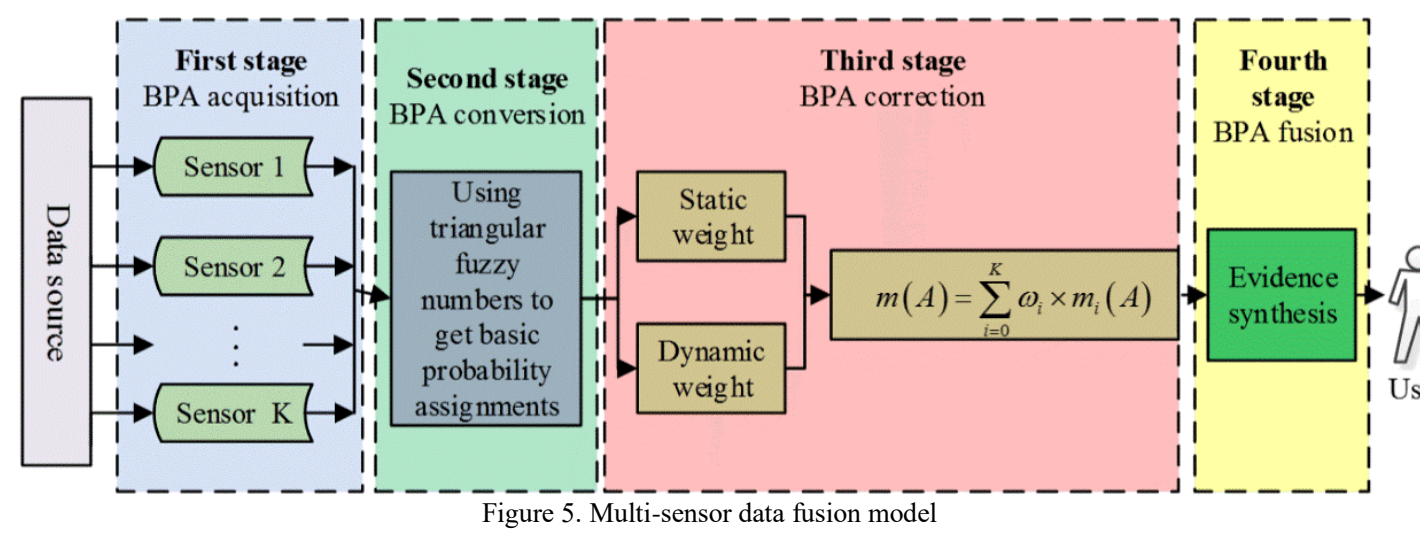

In this hypothesis model, sensor data is pre-processed, the fuzzy membership degree is used to model the data, the sensor reliability is calculated as the weight, the weighted correction BPA is used, and finally the BPA fusion decision is made and the recognition result is obtained.

\section{Simulation}

\subsection{Experiment 1}

The data source is a contest data. The confidence of the three sensors for the target 1 is $0.4,0.8$, and 0.5 , respectively, and the reliability of the three sensors is $0.8,0.7$, and 0.75 , respectively. Because the height data collected by the three sensors is always 0 and the recognition target is the ship, the amount of altitude information collected can be considered as small. In Experiment 1, we ignore the height data feature.

At this point, the three sets of evidence for the three sensors obtained using the evidence modelling method of this scheme are shown in the following table.

Table 1. BPAs of three sensors (ignoring height)

\begin{tabular}{l|c|c}
\hline Sensor type & Target 1 is identifiable & Target 1 is not identifiable \\
\hline 1_AIS interpretation & 0.4903 & 0.5097 \\
\hline 2_Radar detection & 0.6301 & 0.3699 \\
\hline 3_Radar radiation source detection data & 0.9707 & 0.0293 \\
\hline
\end{tabular}

Analyze and observe the data in Table 1. It can be found that the evidence obtained by sensor 1 has very little conflict with the evidence of the other two sensors, that is, inconsistency.

The weighted modified evidence is merged and compared with the traditional D-S fusion method. The results are shown in Table 2.

Table 2. Fusion results of three sensors (ignoring height)

\begin{tabular}{l|c|c}
\hline Fusion method & Target 1 is identifiable & Target 1 is not identifiable \\
\hline D-S fusion method & 0.9819 & 0.0181 \\
\hline Deng entropy fusion method [25] & 0.7835 & 0.2165 \\
\hline This article fusion method & 0.9585 & 0.0415 \\
\hline
\end{tabular}

From the fusion results, we can see that when there is a slight conflict between the evidences, this scheme fusion method can also effectively identify the targets. Deng entropy fusion method is improved effectively, but the recognition rate is slightly lower than the D-S fusion method. The reason for this phenomenon is that this method reduces the 
confidence of conflict evidence and redistributes the weights so that the confidence of valid evidence is slightly reduced. However, the conflict between the experimental data is small, which leads to a decrease in the recognition effectiveness of the fusion method.

\subsection{Experiment 2}

The data is provided in Experiment 1. The confidence of the three sensors for the recognition of the target 1 is $0.4,0.8$, and 0.5 , respectively. The reliability of the three sensors is respectively 0.8, 0.7, and 0.75. Unlike Experiment 1 , Experiment 2 does not ignore the height data feature.

At this point, the three sets of evidence for the three sensors obtained using the evidence modeling method of this scheme are shown in Table 3.

Table 3. BPA of three sensors (without ignoring height)

\begin{tabular}{l|c|c}
\hline Sensor type & Target 1 is identifiable & Target 1 is not identifiable \\
\hline 1_AIS interpretation & 0.7704 & 0.2296 \\
\hline 2_Radar detection & 0.8270 & 0.1730 \\
\hline 3_Radar radiation source detection data & 0.9946 & 0.0054 \\
\hline
\end{tabular}

It can be seen from Table 3 that the three sensors have more consistent evidence information after adding height features.

Table 4. The fusion results of the three sensors (without ignoring the height)

\begin{tabular}{l|c|c}
\hline Fusion method & Target 1 is identifiable & Target 1 is not identifiable \\
\hline D-S fusion method & 0.9997 & 0.0003 \\
\hline Deng Entropy fusion method & 0.9872 & 0.0128 \\
\hline This article fusion method & 0.9964 & 0.0036 \\
\hline
\end{tabular}

From the fusion results, it can be seen that when the evidence is more consistent, the degree of recognition of this scheme fusion method and D-S fusion method is almost close to 1 , which verifies the validity of the fusion model.

\subsection{Analysis of results}

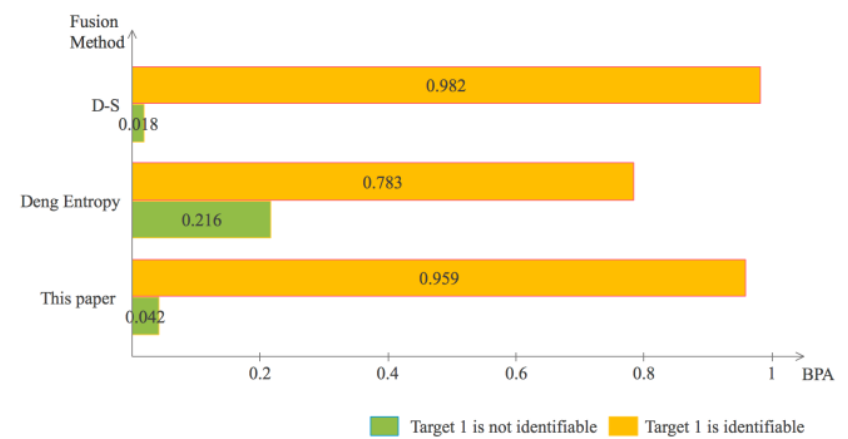

(a)

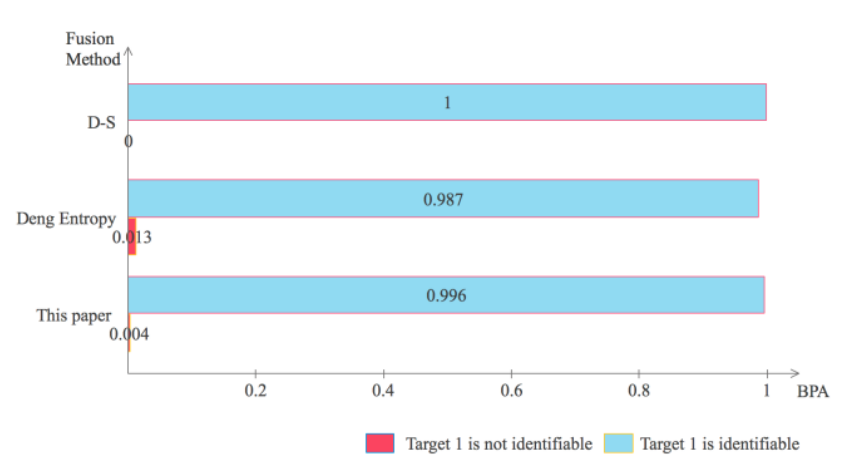

(b)

Figure 6. Experimental results (a) experiment 1 (b) experiment 2

It can be analyzed from the experimental results that in the binary identification problem, the results of the improved fusion method for entropy weights are much better than those of the Deng entropy fusion method.

Both the D-S fusion method and the method fusion method can achieve the recognition of the target 1; even if the D-S fusion method is in the case of slight conflict, the fusion result is better than the fusion result of the present solution. There are two reasons for this result.

The conflict between the data in the database is relatively small; the data modeling method of this program effectively maintains the relationship between the original data, so the conflict between the evidence obtained is also relatively small, and there is no conflict.

The improved fusion algorithm of this paper is mainly focused on the problem of D-S fusion failure when $\mathrm{k}$ is close to 1 
when there is high conflict between the evidences. The so-called gains and losses, reducing the confidence of conflicting evidence will also affect the confidence of normal evidence; but, this method is still very effective in dealing with conflicts.

\section{Conclusions}

Evidence theory, as the basic theory of fusion of this model, is an effective tool and method to deal with and express the uncertainty problem. The multi-sensor information fusion algorithm based on D-S evidence theory does not need to specify the prior probability, and can effectively solve the fusion problem caused by uncertainty information between sensors. Using triangle fuzzy numbers for evidence modelling, compared with other evidence modelling methods, the calculation amount is small, the calculation time and cost are saved, and it also has a good modelling effect. In this paper, aiming at the binary identification problem, the irrationality of the traditional Deng entropy weight is modified to improve the accuracy of the weighted fusion method in the binary identification problem. However, the improved weighted fusion method of entropy weights can only be used for the binary identification problem, which has a large limitation. The model uses multiple sensors to confirm the same target event from different sides, improving the reliability, and robustness of the detection.

Technical factors and noise have effect on sensors, such as technical principles, materials, manufacturing processes, etc. Therefore, the static reliability of the sensor is an important factor to measure the reliability of the sensor. On the other hand, because the reliability of the sensor is also connected to the target and surrounding properties, like environmental noise, the existence of an unknown target, and the deceptive behavior of the observation target, etc. The dynamic reliability of the sensor is also an important factor to measure the reliability of the sensor. This paper assumes that the product of evidence distance and Deng entropy is the dynamic reliability of the sensor. The product modelling of the final static reliability and dynamic reliability is assumed to be the overall reliability of the sensor. The rationality of this modelling method is that it takes into account the internal factors of the sensor and combines with the external environment, thereby reducing the impact of objective factors on the final recognition fusion decision.

\section{References}

1. B. Cai, Y. Zhao, H. Liu, and M. Xie, "A data-driven fault diagnosis methodology in three-phase inverters for PMSM drive systems," IEEE Transactions on Power Electronics, vol. 32, no. 7, pp. 5590-5600, July 2017

2. B. Chen, J. Wang, and S. Chen, "Prediction of pulsed GTAW penetration status based on BP neural network and DS evidence theory information fusion," The International Journal of Advanced Manufacturing Technology, vol. 48, no. 1-4, pp. 83-94, April 2010

3. M. Delgado, J. A. C. Corrales, J. J. Saucedo-Dorantes, R. D. J. Romero-Troncoso and R. A. A. Osornio-Rios, "Thermography based Methodology for Multi-fault Diagnosis on Kinematic Chain[J]," IEEE Transactions on Industrial Informatics, pp. 1-1, March 2018

4. Y. Deng, "Deng Entropy: A Generalized Shannon Entropy to Measure Uncertainty", (online since January 2015) (DOI: 10.5281/zenodo.32211)

5. G. Dong and G. Kuang, "Target Recognition via Information Aggregation Through Dempster-Shafer's Evidence Theory[J]," IEEE Geoscience and Remote Sensing Letters, vol. 12, no. 6, pp. 1247-1251, February 2015

6. D. Grießbach, D. Baumbach, A. Börner, M. Buder, I. Ernst, E. Funk, J. Wohlfeil and S. Zuev, "IPS-A system for real-time navigation and 3D-modeling," International Archives of the Photogrammetry, Remote Sensing and Spatial Information Sciences (ISPRS), pp. 21-26, Melbourne, Australia, July 2012

7. H. Guo, W. Shi and Y. Deng, "Evaluating sensor reliability in classification problems based on evidence theory," IEEE Transactions on Systems Man \& Cybernetics Part B Cybernetics A Publication of the IEEE Systems Man \& Cybernetics Society, vol. 36, no. 5, pp. 970-981, November 2006

8. T. Horiuchi, "Decision rule for pattern classification by integrating interval feature values," IEEE Transactions on Pattern Analysis and Machine Intelligence, vol. 20, no. 4, pp. 440-448, April 1998

9. A. L. Jousselme, D. Grenier and É. Bossé, "A new distance between two bodies of evidence," Information fusion, vol. 2, no. 2, pp. 91-101, June 2001

10. H. Li, J. Zhao, X. Zhang, and X. Ni, "Fault Diagnosis for Machinery based on Feature Selection and Probabilistic Neural Network," International Journal of Performability Engineering, vol. 13, no. 7, pp. 1165-1170, November 2017

11. Y. Lin, C. Wang, C. Ma, Z. Dou, and X. Ma, "A new combination method for multisensor conflict information," Journal of Supercomputing, vol. 72, no. 7, pp. 2874-2890, March 2016

12. Y. Lin, X. Zhu, Z. Zheng, Z. Dou, and R. Zhou, "The individual identification method of wireless device based on dimensionality reduction and machine learning," Journal of Supercomputing, no. 5, pp. 1-18, December 2017

13. K. Ma, H. Li, H. Yong, Z. Wang, D. Meng and L. Zhang, "Robust multi-exposure image fusion: A structural patch decomposition approach[J]," IEEE Transactions on Image Processing, vol. 26, no. 5, pp. 2519-2532, February 2017

14. C. U. Mba, H. A. Gabbar, S. Marchesiello, A. Fasana, and L. Garibaldi, "Fault Diagnosis in Flywheels: Case Study of a Reaction Wheel Dynamic System with Bearing Imperfections," International Journal of Performability Engineering, vol. 13, no. 4, pp. 362-373, July 2017

15. E. Ristani, and C. Tomasi, "Features for Multi-Target Multi-Camera Tracking and Re-Identification," Computer Vision and 
Pattern Recognition, Mar 2018

16. C. Shi, Z. Dou, Y. Lin, and W. Li, "Dynamic threshold-setting for RF-powered cognitive radio networks in non-Gaussian noise," Physical Communication, vol. 27, pp. 99-105, April 2018

17. G. Wang, N. Li, and Y. Zhang, "An event based multi-sensor fusion algorithm with deadzone like measurements," Information Fusion, vol. 42, pp. 111-118, July 2018

18. L. Yan, Y. Lu, and Y. Zhang, "An improved NLOS identification and mitigation approach for target tracking in wireless sensor networks," IEEE Access, vol. 5, no. 99, pp. 2798-2807, March 2017

19. H. Yin, "Tensor Sparse Representation for 3-D Medical Image Fusion Using Weighted Average Rule[J]," IEEE Transactions on Biomedical Engineering, pp. 1-1, February 2018

20. K. Yuan, F. Xiao, L. Fei, B. Kang, and Y. Deng, "Modeling sensor reliability in fault diagnosis based on evidence theory," Sensors, vol. 16, no. 1, pp. 113, January 2016

21. L. A. Zadeh, "Review of a mathematical theory of evidence," AI magazine, vol. 5, no. 3, pp. 235-247, September 1984

22. X. Zhang, F. Zhao, and J. Kang, "Case Studies for Bearing Fault Diagnosis based on Adaptive Myriad Filter and Alpha Stable Model," International Journal of Performability Engineering, vol. 13, no. 4, pp. 551-555, July 2017

23. W. Zhao, H. Lu and D. Wang, "Multisensor image fusion and enhancement in spectral total variation domain[J]," IEEE Transactions on Multimedia, vol. 20, no. 4, pp. 866-879, October 2018 